\title{
Emission of dispersive waves from solitons in axially-varying optical fibers
}

\author{
A. Kudlinski, A. Mussot, M. Conforti and D. V. Skryabin
}

\begin{abstract}
The possibility of tailoring the guidance properties of optical fibers along the same direction as the evolution of the optical field allows to explore new directions in nonlinear fiber optics. The new degree of freedom offered by axiallyvarying optical fibers enables to revisit well-established nonlinear phenomena, and even to discover novel short pulse nonlinear dynamics. Here we study the impact of meter-scale longitudinal variations of group velocity dispersion on the propagation of bright solitons and on their associated dispersive waves. We show that the longitudinal tailoring of fiber properties allows to observe experimentally unique dispersive waves dynamics, such as the emission of cascaded, multiple or polychromatic dispersive waves.
\end{abstract}

\section{Introduction}

Since its discovery in the frame of the Korteweg-de Vries equation by Zabusky and Kruskal [1, 2], the concept of solitons has been extended to many other systems described by integrable equations [3], including the nonlinear Schrödinger equation (NLSE) [4] widely used to study nonlinear pulse propagation in optical fibers [5, 6, 7]. However, in real-world fibers, intrinsic higher-order dispersive and nonlinear effects break the integrability of the NLSE and therefore perturb the invariant propagation of solitons [8]. Fundamental solitons, on the contrary to higher-order ones, are very stable in optical fibers and they usually survive these perturbations, by continuously adapting their temporal and spectral shapes [9]. The robustness of

A. Kudlinski, A. Mussot, M. Conforti

Univ. Lille, CNRS, UMR 8523 - PhLAM - Physique des Lasers Atomes et Molécules, F-59000

Lille, France, e-mail: alexandre.kudlinski@univ-lille1.fr

D. V. Skryabin

Department of Physics, University of Bath, Bath, United Kingdom e-mail: d.v.skryabine

bath.ac.uk 
fundamental solitons has been exploited in the context of dispersion-managed optical communications in which the periodic evolution of losses and gain due to fiber attenuation and amplifiers is compensated by a periodic arrangement of dispersion and/or nonlinearity [10]. The so-called dispersion-managed solitons are propagating over kilometer-long systems and they are usually relatively broad (in the picosecond duration scale), making them weakly altered by higher-order dispersion. In fact, in some cases, third-order dispersion might even help to stabilize them [11]. In the case of much narrower solitons (in the hundreds of femtoseconds time scale), higher-order dispersion plays a much more significant role. In the case of a perturbation due to third-order dispersion for example, a short soliton propagating near the zero-dispersion wavelength (ZDW) loses energy into a dispersive wave (also called resonant or Cherenkov radiation) across the ZDW [12, 13] and experiences a spectral recoil in the opposite spectral direction in order to conserve the overall energy [14]. This very well-known process has been studied extensively from theoretical and experimental points of views [12, 13, 14, 15, 16, 17, 18], in particular in the context of supercontinuum generation in which it plays a crucial role in the early dynamics [7, 19]. Following their emission, dispersive waves can also collide with solitons in presence of Raman effect, leading to their nonlinear interaction [20, 21, 22]. Understanding this nonlinear wave mixing process has been a key in extending supercontinuum sources towards the blue/ultraviolet spectral region [19, 23, 24] and also towards long wavelengths in some specific cases [25].

In this chapter, we study the process of dispersive wave emission from a soliton in various axially-varying optical fibers. We show that the longitudinal variation of guiding properties allows observe experimentally new and unique dynamics regarding dispersive waves. The first section introduces the basics of dispersive wave emission from a fundamental soliton and the second one focuses on the peculiarities of this process in axially-varying fibers.

\section{Emission of a dispersive wave from a soliton}

In this section, we will introduce the basic concepts of fundamental temporal soliton propagation in optical fiber with second-order dispersion [or group-velocity dispersion (GVD)] and Kerr nonlinearity, as well as radiation of dispersive wave in presence of third-order dispersion.

\section{Fundamental soliton}

The nonlinear propagation of light in dispersive and nonlinear optical fibers is described by the nonlinear Schrödinger equation (NLSE) 


$$
\frac{\partial A}{\partial z}=-i \frac{\beta_{2}}{2} \frac{\partial^{2} A}{\partial^{2} t}+i \gamma|A|^{2} A
$$

Here, $t$ is the retarded time in the frame traveling at the group velocity $v_{g}$ of the input pulse. $A(z, t)$ is the envelope of the electric field, $\beta_{2}$ is the fiber GVD coefficient and $\gamma$ is the Kerr nonlinear parameter defined using the standard definition from [26]. The fundamental soliton is an analytical solution of Eq. [1] [4] when the second-order dispersion coefficient is negative (anomalous GVD) and the nonlinear parameter $\gamma$ is positive. Its amplitude takes the form

$$
A(z, t)=\sqrt{P_{0}} \operatorname{sech}\left(\frac{t}{T_{0}}\right)
$$

where $P_{0}$ is the soliton peak power and $T_{0}$ its duration. The soliton is termed fundamental when the following condition is fulfilled

$$
P_{0}=\frac{\left|\beta_{2}\right|}{\gamma T_{0}^{2}}
$$

Figure 1 shows the results of a numerical integration of Eq. 1 in a standard telecommunication single-mode fiber. The second-order dispersion coefficient is $\beta_{2}=-6.05 \times 10^{-28} \mathrm{~s}^{2} / \mathrm{m}$ and the nonlinear parameter is $\gamma=2.08 \mathrm{~W}^{-1} . \mathrm{km}^{-1}$. The input pulse is a fundamental soliton centered at $1310 \mathrm{~nm}$ with a duration $T_{0}$ of 100 fs and a peak power of $29.08 \mathrm{~W}$ (in accordance with Eq. 3). Figures 1 a, a,b) and (c,d) show that the input pulse propagates without any spectral or temporal modification along the fiber, respectively. This is the most striking feature of temporal fundamental solitons and this is the main reason why they have attracted so much interest both from fundamental and applicative point of views [6, 27, 28].

\section{Dispersive wave}

In practice however, one cannot always neglect the fiber third-order dispersion. In particular, it plays a major role when the soliton is located near the zero-dispersion wavelength (ZDW) of the fiber [12, 13], i.e. when the soliton spectrum overlaps the normal GVD region located across the ZDW. In this case, Eq. 1 has to be rewritten in order to take into account the fiber third-order dispersion. It takes the form

$$
\frac{\partial A}{\partial z}=-i \frac{\beta_{2}}{2} \frac{\partial^{2} A}{\partial^{2} t}+\frac{\beta_{3}}{6} \frac{\partial^{3} A}{\partial^{3} t}+i \gamma|A|^{2} A
$$

where $\beta_{3}$ is the third-order dispersion coefficient of the fiber. The third-order dispersion drastically modifies the soliton dynamics as compared to the case in which it is neglected. This is illustrated in Fig. 2 2 where we have simulated the propagation of the same input pulse as above using Eq. 4. We have considered a $\beta_{3}$ value of $6.9 \times 10^{-41} \mathrm{~s}^{3} / \mathrm{m}$, the other fiber parameters being the same ones as above. The fiber 
ZDW [represented by the black dotted line in Figs 2 a) and (b)] is $1302 \mathrm{~nm}$. The output spectrum obtained after $200 \mathrm{~m}$ [red solid line in Fig. 2(a)] strongly differs from the input one (black dashed line). First, it exhibits a strong peak around 1280 $\mathrm{nm}$ corresponding to a dispersive wave. Second, the soliton spectrum is distorted and its central wavelength has red shifted from $1310 \mathrm{~nm}$ to $1313 \mathrm{~nm}$. This is the spectral recoil accompanying the emission of the dispersive wave. The spectral dynamics displayed in Fig. 2(b) shows that the spectrum initially broadens towards the short-wavelength region and that the dispersive wave starts to form at about $50 \mathrm{~m}$ of propagation. In the time domain [Fig. 2 (2) ], it appears at the trailing edge of the soliton (red solid line) as a broad and relatively distorted pulse (see inset). Since the soliton has experienced a spectral recoil, it has slightly decelerated as compared to the fiber input. It has also lost some energy, which has been radiated into the dispersive wave. This can be further observed in the temporal dynamics plot of Fig. 2 (d), where we can see the slow deceleration of the soliton as well as the continuous radiation of a broad dispersive wave along propagation.

The frequency at which the dispersive wave is emitted can be predicted from phase-matching arguments between the two radiations as [14]
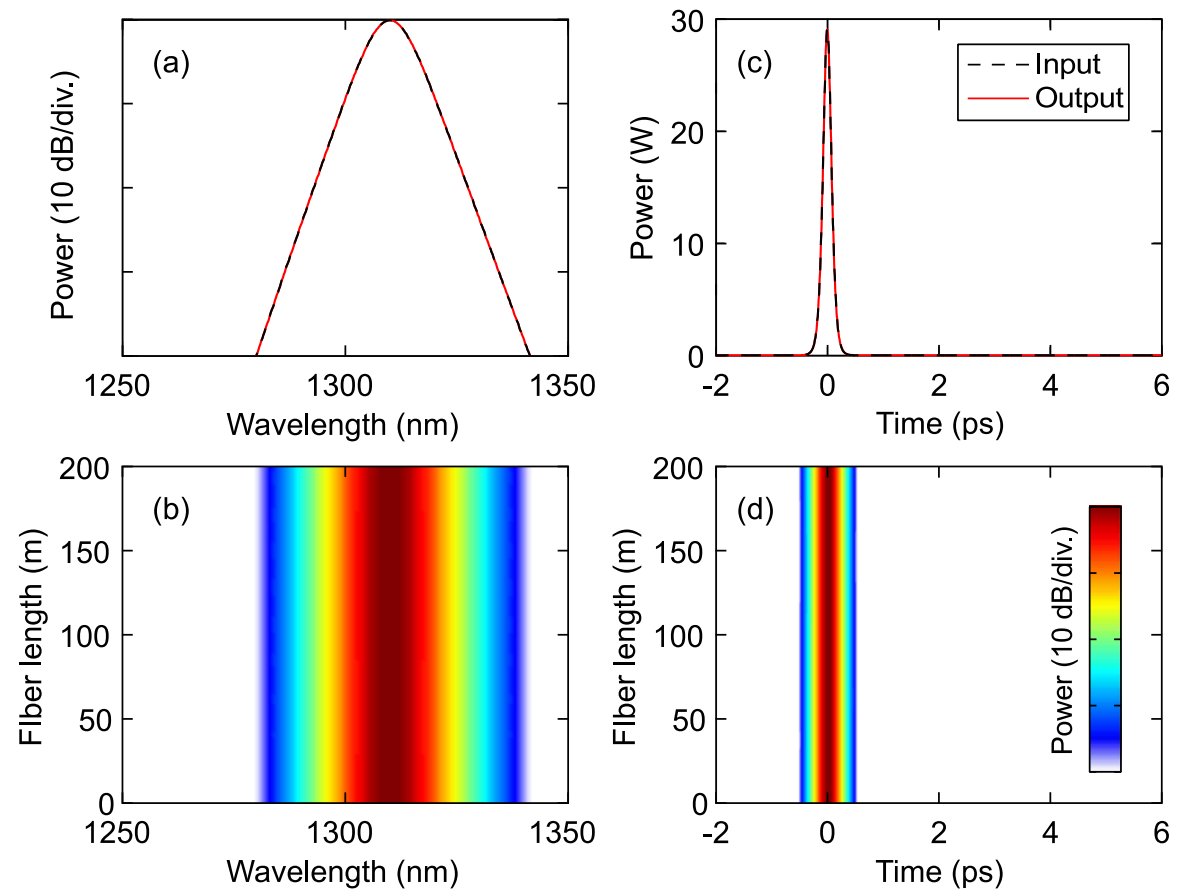

Fig. 1 Simulation of soliton propagation using Eq. 1 i.e. neglecting third-order dispersion. (a) Input (black dashed line) and output (red solid line) spectra in a standard telecommunication singlemode fiber of $200 \mathrm{~m}$ length. (b) Spectral dynamics along propagation, with the same colorscale as in (d). (c) Corresponding input (black dashed line) and output (red solid line) temporal profiles. (d) Temporal dynamics along propagation. Simulation parameters are given in the text. 

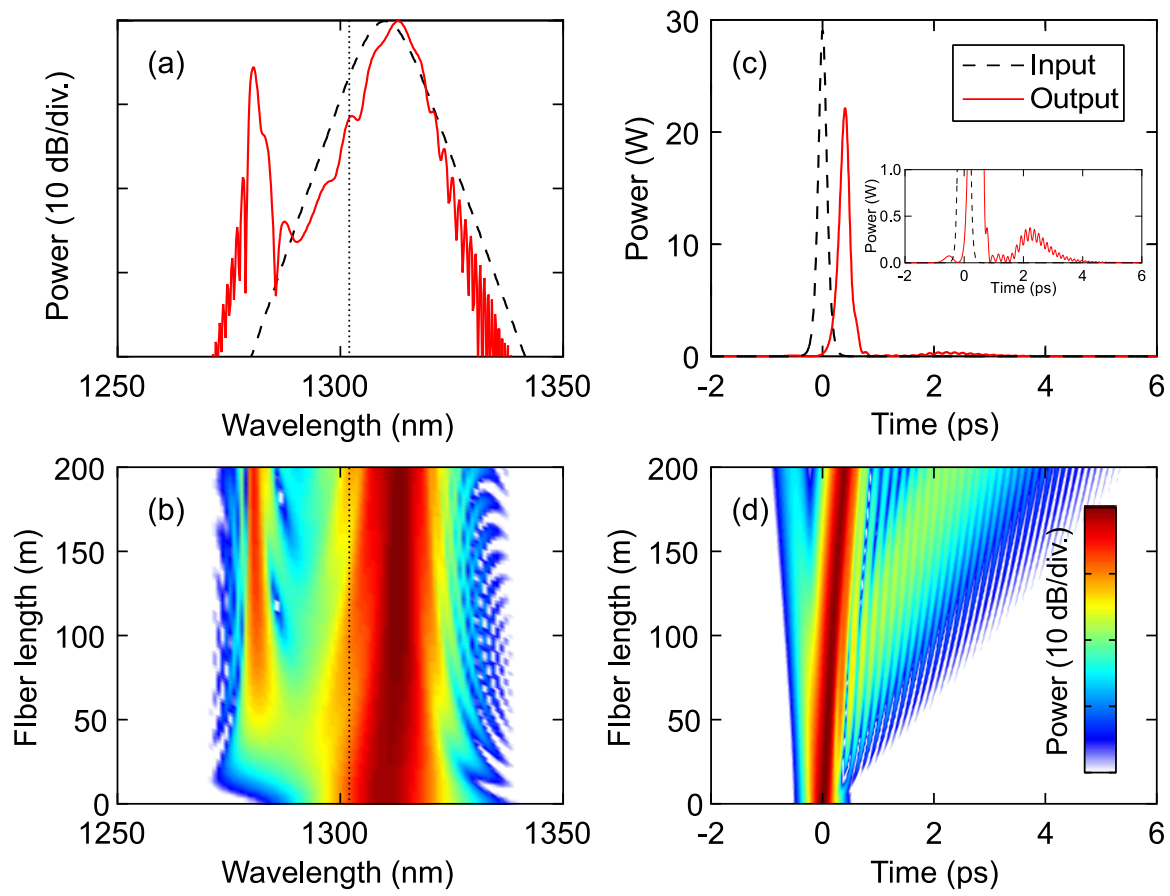

Fig. 2 Simulation of soliton propagation using Eq. 4 i.e. taking third-order dispersion into account. (a) Input (black dashed line) and output (red solid line) spectra in a standard telecommunication single-mode fiber of $200 \mathrm{~m}$ length. (b) Spectral dynamics along propagation, with the same colorscale as in (d). (c) Corresponding input (black dashed line) and output (red solid line) temporal profiles. (d) Temporal dynamics along propagation. Simulation parameters are given in the text. The black dotted lines in (a) and (b) represent the fiber ZDW.

$$
\frac{\beta_{2}}{2} \Omega^{2}+\frac{\beta_{3}}{6} \Omega^{3}=\frac{\gamma P_{0}}{2}
$$

where $\Omega=\omega_{S}-\omega_{D W}$ is the frequency separation between the soliton at $\omega_{S}$ and the dispersive wave at $\omega_{D W}$. Solving Eq. 5 with the parameters of our study gives a dispersive wave wavelength of $1283 \mathrm{~nm}$, in excellent agreement with the numerical simulation results of Figs 2 a) and (b). The process of dispersive wave emission from a soliton is very well known and explained as long as optical fibers are uniform in length: when the soliton spectrum crosses the ZDW, the part of soliton energy located in the normal GVD region is radiated into a dispersive wave. This causes a spectral recoil of the soliton which thus moves away from the ZDW so that the emission process becomes less and less efficient and cannot occur again. Consequently, there is a unique dispersive wave which is generated and appears as a sharp spectral peak whose frequency does not change with propagation, as observed from Fig. 2.b). However, the process can be radically different in optical fibers which are not uniform as a function of length, i.e. in axially-varying fibers. This will be the focus of the remaining of this chapter. 


\section{Generation of dispersive waves from solitons in axially-varying optical fibers}

\section{Axially-varying optical fibers}

For the fabrication of axially-varying optical fibers, the longitudinal evolution of the fiber diameter is controlled by adjusting the evolution of drawing speed with time (which is related to fiber length) using a servo-control system. This process is ruled by the conservation of glass mass between the preform and the fiber:

$$
d_{\text {Fiber }}=d_{\text {Preform }} \sqrt{\frac{V_{\text {Preform }}}{V_{\text {Fiber }}}}
$$

where $d_{\text {Preform,Fiber }}$ are respectively the preform and fiber outer diameter, and $V_{\text {Preform,Fiber }}$ are respectively the preform feed into the furnace and the drawing capstan speed. In our process, $d_{\text {Preform }}$ and $V_{\text {Preform }}$ are fixed, $V_{\text {Fiber }}$ is adjusted with a desired $f(z)$ function (where $z$ is the longitudinal space coordinate along the fiber), which results in a modulation of the fiber outer diameter $d_{\text {Fiber }}(z)$, and thus of the overall fiber structure. This results in a modulation of the mode(s) propagation constant(s), and thus of all guiding properties.

Figure 3 (a) shows three examples of axially-varying fibers used hereafter. These curves show the evolution of the outer diameter recorded during the fiber drawing process. Figure 3(b) shows the corresponding calculated ZDW. The example represented in red corresponds to a fiber with two ZDWs, and the plot in Fig. 3. b) corresponds to the second ZDW. In the range of diameter variations investigated here, the ZDW approximately follows a linear dependance with fiber diameter.

\section{Emission of multiple dispersive waves along the fiber}

As recalled above, in uniform fibers, the soliton experiences a red shift (spectral recoil) during the generation of a dispersive wave. This red shift is usually strongly reinforced by the Raman-induced soliton self-frequency shift. As a consequence, the soliton moves far away from the ZDW, so that the dispersive wave emission process cannot occur again. We will first show an example in which the use of a suitable axially-varying fiber allows for a single soliton to emit multiple dispersive waves along the fiber. Indeed, this can occur if the fiber ZDW moves along propagation so that it "hits" the soliton during its redshift following the emission of a dispersive wave [29, 30]. In this case, the overlap between the soliton spectrum and the normal GVD region can become large enough to initiate the emission of a new dispersive wave. This is illustrated with numerical simulations and experiments in Figs. 4(a) and (b), respectively. For these numerical simulations, the stimulated Raman scattering term has been added to Eq. 4, which now writes: 

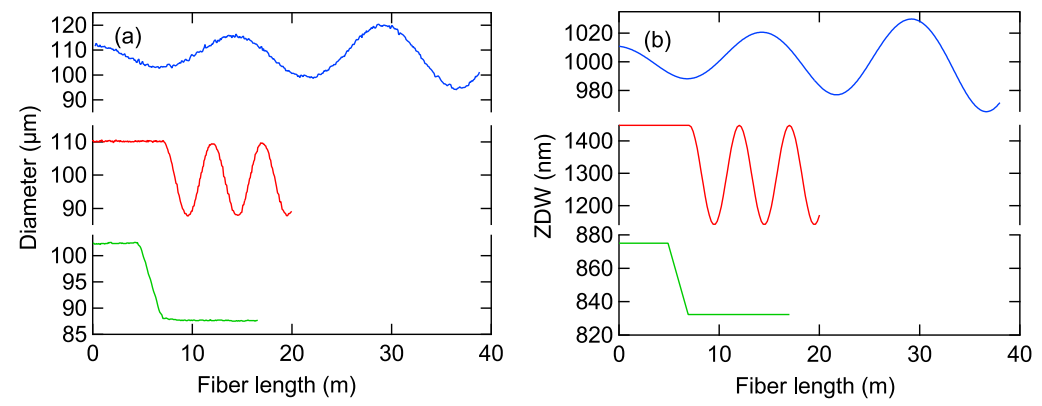

Fig. 3 (a) Evolution of the outer diameter as a function of fiber length for three different axiallyvarying fibers measured during the fiber fabrication process. (b) Corresponding calculated ZDW. For the second fiber (red case), the ZDW plotted in (b) corresponds to the second one (located at long wavelengths). 


$$
\frac{\partial A}{\partial z}=-i \frac{\beta_{2}}{2} \frac{\partial^{2} A}{\partial^{2} t}+\frac{\beta_{3}}{6} \frac{\partial^{3} A}{\partial^{3} t}+i \gamma\left(A \int R\left(t^{\prime}\right)\left|A\left(t-t^{\prime}\right)\right|^{2} d t^{\prime}\right)
$$

where $R(t)=\left(1-f_{R}\right) \delta(t)+f_{R} h_{R}(t)$ includes both Kerr and Raman effects, where $h_{R}(t)$ correspond to the Raman response function $\left(f_{R}=0.18\right)$ taken from [31].

We consider a fiber with a ZDW which evolves along the fiber [blue curve in Figs. [3(a) and (b)], following the profile represented by the black solid lines in Fig. 4 (a) and (b). The ZDW is $1011 \mathrm{~nm}$ at the fiber input and reaches $1021 \mathrm{~nm}$ and $1030 \mathrm{~nm}$ at $14 \mathrm{~m}$ and $29 \mathrm{~m}$, respectively. Simulations and experiments are performed with an input pulse of $410 \mathrm{fs}$ full width at half maximum (FWHM) duration centered around $1030 \mathrm{~nm}$ and a peak power of $46 \mathrm{~W}$. In order to be consistent with experiments, we consider a slight chirp with a chirp parameter of +3.7 . All parameters can be found in Ref. [30]. The simulation in Fig. 4(a) shows the emission of a first dispersive wave around $925 \mathrm{~nm}$ at a length of $5 \mathrm{~m}$, in a similar fashion to what happens in uniform fibers (see Fig. 2). The soliton experiences a red shift due to the combined action of spectral recoil and Raman effect. The ZDW increases to $1021 \mathrm{~nm}$ at 14 $\mathrm{m}$, which brings it closer to the soliton and therefore enhances the spectral overlap between the soliton and the normal GVD region. This initiates the emission of a second dispersive wave around $960 \mathrm{~nm}$ at this location in the fiber. The same process occurs a third time around $29 \mathrm{~m}$, resulting in the emission of a third dispersive wave. Experiments reported in Fig. 4(b) are in excellent agreement with simulations and demonstrate the process of multiple dispersive wave emission from a single soliton.
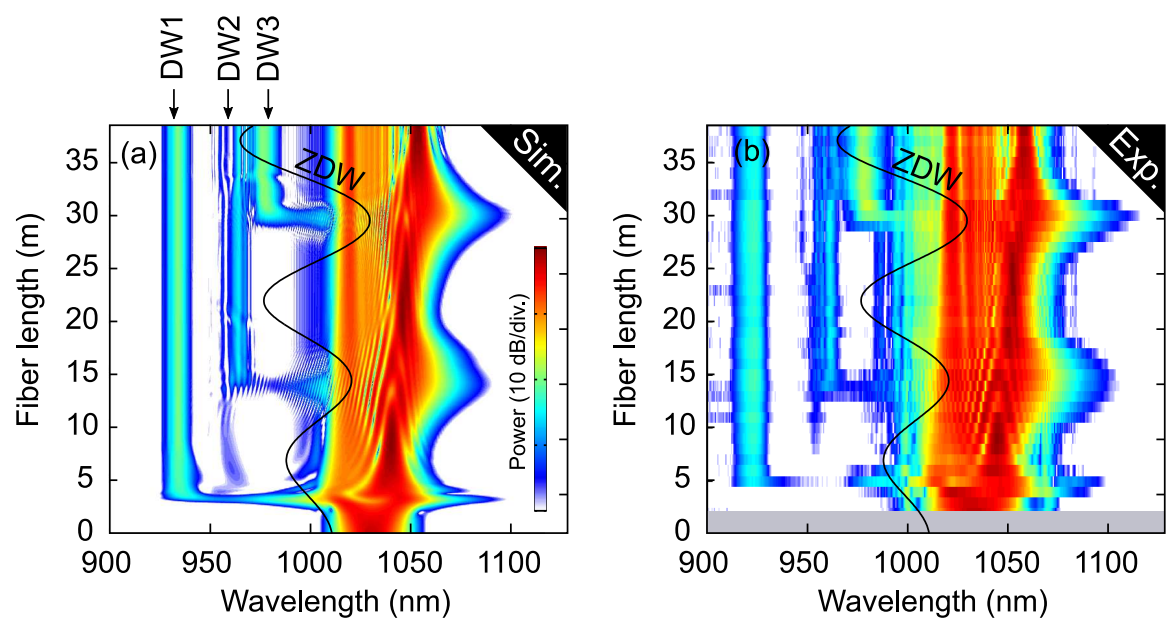

Fig. 4 (a) Numerical simulations and (b) experimental results in the spectral domain versus fiber length showing the emission of three distinct dispersive waves from a single soliton in an axiallyvarying fiber. The evolution of the ZDW with fiber length is represented by black solid lines. 


\section{Cascading of dispersive waves}

The process of dispersive wave emission can also occur in a slightly different scenario in uniform fibers: a soliton experiencing a strong Raman-induced selffrequency shift can hit the second ZDW of a fiber (located at long wavelengths), which cancels the red shift and generates a dispersive wave across the ZDW, at longer wavelengths [32, 33]. Here we will study this process in an axially-varying fiber [red curve in Figs. 3(a) and (b)] with two ZDWs separated by a region of anomalous dispersion in which a fundamental soliton is excited. The evolution of the second ZDW (the long wavelength one) is represented by the white solid lines in Figs. 5. It is constant over the first $7 \mathrm{~m}$ and then oscillates as a function of length. We consider input pulses of $340 \mathrm{fs}$ FWHM duration centered around $1030 \mathrm{~nm}$, with a chirp parameter of +1.5 and a peak power of $75 \mathrm{~W}$. Figures 5 (a) and (b) show respectively the numerical simulations performed with Eq. 7 and experimental results.

The input short pulse excites a fundamental soliton which initially experiences Raman-induced soliton self-frequency shift. Around $9 \mathrm{~m}$, the ZDW has reached $1140 \mathrm{~nm}$ and is very close to the soliton so that a dispersive wave [labelled DW1 in Fig. [5.a)] is emitted across the ZDW, in the normal GVD region. At this point, the process is very similar to the one known in uniform fibers. The real novelty comes slightly after $10 \mathrm{~m}$, when DW1 crosses the ZDW (which is increasing again at this point): as soon as DW1 crosses the ZDW, a new radiation (labelled CDW1) is generated at even longer wavelengths, around $1350 \mathrm{~nm}$. A careful analysis of this process reveals that the continuously evolving GVD prevent the dispersive wave to strongly spread out in time [as expected in uniform fibers, see Fig. 2[d)] and allows to keep it relatively localized in time as a short pulse [34]. As a consequence, when crossing the ZDW, this pulse can emit another dispersive wave which we term cascaded dispersive wave (CDW1), in analogy with cascaded four-wave mixing processes.

The exact same process occurs again farther in the fiber, around $15 \mathrm{~m}$. The ZDW decreases again very close to the remaining of the soliton located slightly above $1100 \mathrm{~nm}$ so that a dispersive wave, DW2, is emitted. When DW2 crosses the ZDW slighty after, a cascaded dispersive wave, CDW2, is emitted. Experiments reported in Fig. 5. (b) show again excellent agreement with numerical results, and provide the evidence for the process of cascaded dispersive wave generation. We might also note that, similarly to the previous section, multiple dispersive waves (DW1 and DW2) have been observed from a single soliton, around the second ZDW.

\section{Transformation of a dispersive wave into a fundamental soliton}

As mentioned above and deeply analyzed in Ref. [34], the cascaded dispersive wave process is due to the fact that the dispersive wave initially generated experiences a GVD that varies with length, which allows it to remain temporally localized as a pulse to initiate the generation of a cascaded dispersive wave. Here, we will study 

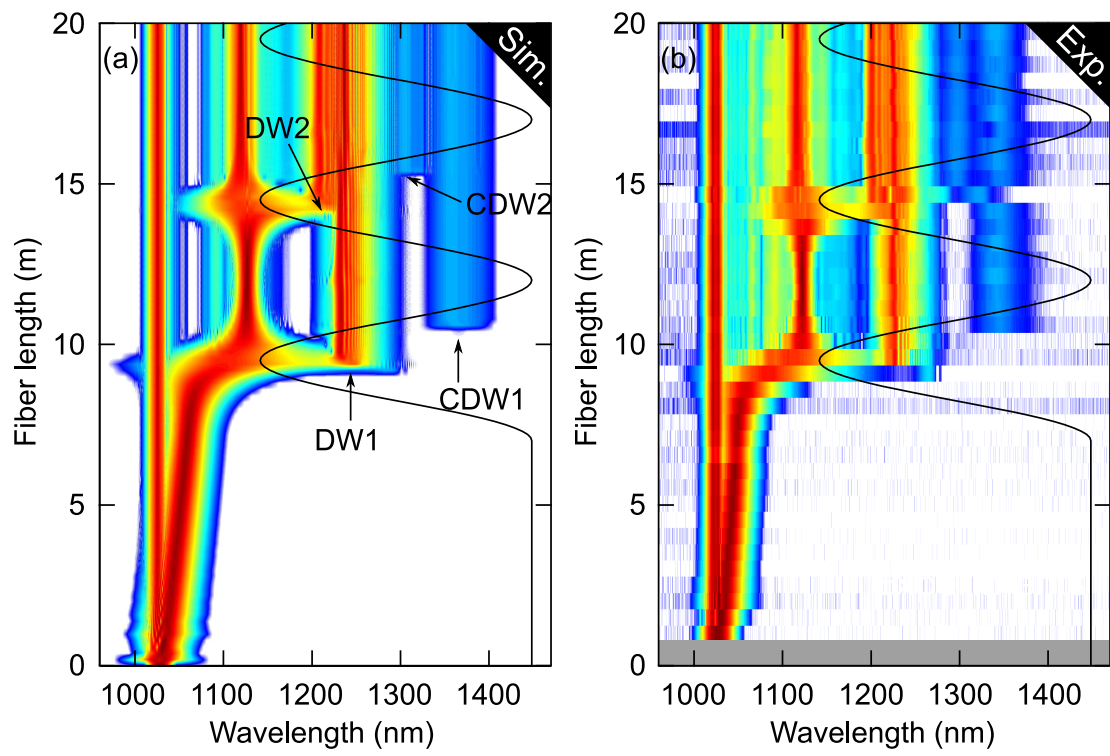

Fig. 5 (a) Numerical simulations and (b) experimental results in the spectral domain versus fiber length showing the emission of two cascaded dispersive waves in an axially-varying fiber with two ZDWs. The evolution of the second ZDW (located at long wavelengths) with fiber length is represented by black solid lines.

this process more into details. For that, we consider a soliton launched in the vicinity of the ZDW so that it emits a dispersive wave in the normal GVD region, similarly to the usual case illustrated in Fig. 2. Once the dispersive wave is emitted, the fiber parameters are changed so that the GVD at the dispersive wave wavelength becomes anomalous [green curve in Figs. 3(a) and (b)] and the evolution of the radiation is studied. The results are summarized in Fig. 6. The input pulse is transform limited with a $140 \mathrm{fs}$ FWHM duration. It is centered around $881 \mathrm{~nm}$ and has a peak power of $42 \mathrm{~W}$. The spectral evolution versus fiber length [simulations in Fig. 6.a) and experiments in Fig.6. (c)] shows the emission of a dispersive wave around $840 \mathrm{~nm}$ but does not highlight much change after the GVD change occurring between 5 and $7 \mathrm{~m}$. The simulated time domain simulation of Fig. 6.b) provides much more information about the dynamics. The soliton decelerates due to the combined effects of spectral recoil and Raman-induced self-frequency shift and the dispersive wave, which starts spreading out, is located slightly behind it, as expected. Once it enters the tapered region (materialized by the two dashed lines), the dispersive wave accelerates due to changing dispersion and crosses the soliton. At the same time, it recompresses and further propagated as a short pulse in the remaining of the fiber, recalling the behavior of a soliton. In fact, further theoretical analysis reveals that the dispersive wave has indeed been transformed into a fundamental soliton [35] and propagates as such in the remaining anomalous dispersion region. Experimental autocorrelation 
measurements reported in Fig.6(d) confirm this behavior: the pulse duration initially increases and then decreases after the tapered region, where they are perfectly fitted by square hyperbolic secant functions [35]. These results show that a dispersive wave emitted from a soliton can be itself transformed into a fundamental soliton by carefully varying the longitudinal evolution of dispersion.

\section{Emission of polychromatic dispersive waves}

In the previous section, we have shown that a dispersive pulse initially travelling in normal GVD can become transformed into a fundamental soliton when entering a
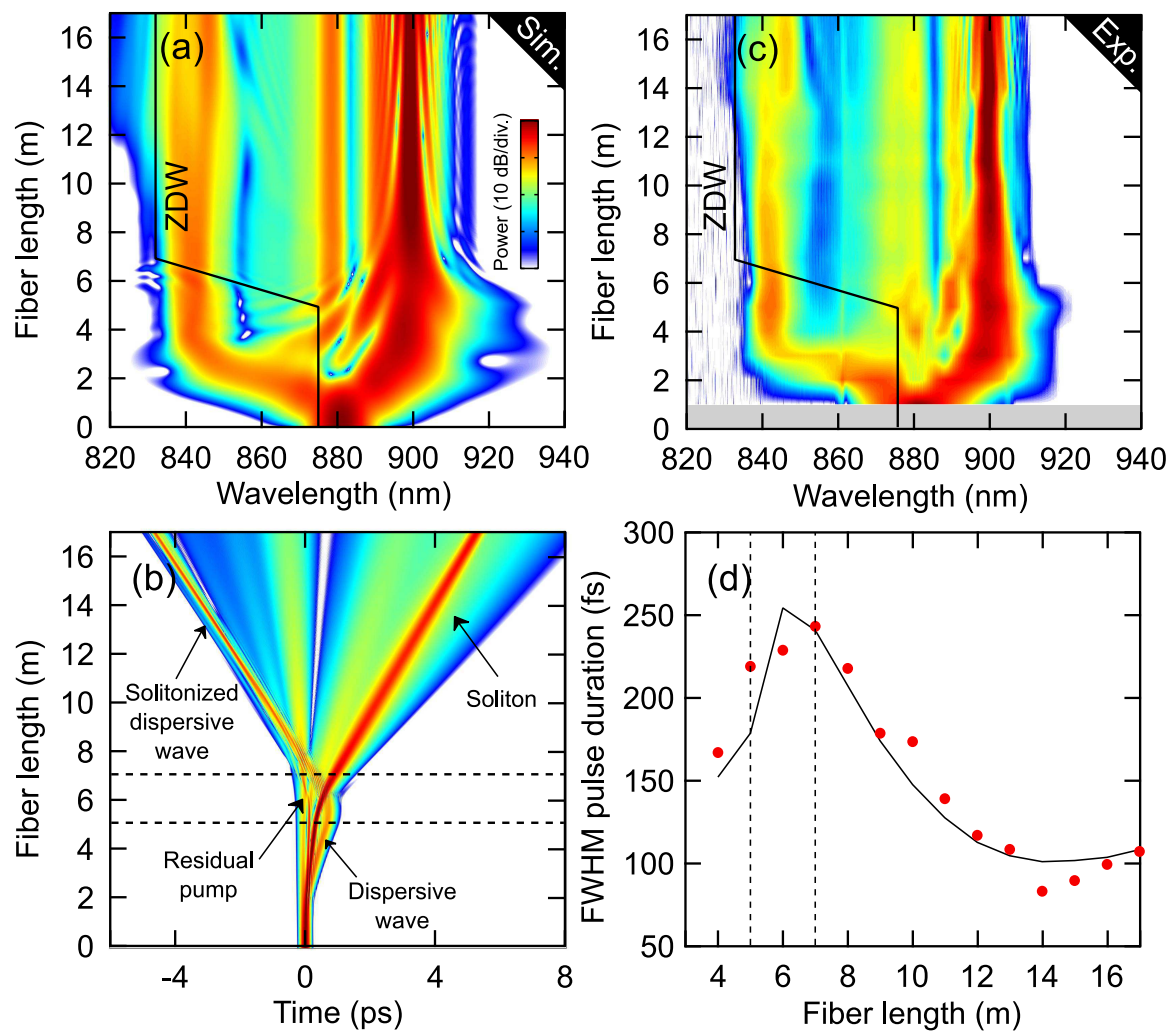

Fig. $6(a, b)$ Numerical simulations and $(c, d)$ experimental results showing the transformation of a dispersive wave into a fundamental soliton. (a) and (b) correspond respectively to the simulated spectral and temporal evolutions. (c) Measured spectral evolution versus fiber length. (d) Measured pulse duration around $840 \mathrm{~nm}$ (red full circles) and simulated one (black solid line). Black solid lines in (a) and (c) depict the ZDW. Dashed lines in (b) and (d) depict the limits of the tapered section. 
region with anomalous dispersion. Here we will study a reversed situation in which a fundamental soliton propagating in anomalous dispersion enters a region with normal dispersion. Fundamentally, the pulse cannot be a soliton anymore, and it is expected to linearly disperse. We will see that it can excite a so-called polychromatic dispersive wave [36, 37].

Here, a fundamental soliton is excited by launching a transform limited pulse with a $130 \mathrm{fs}$ FWHM duration around $950 \mathrm{~nm}$, with a peak power of $110 \mathrm{~W}$. The fiber is uniform over the first $4.5 \mathrm{~m}$ and then it is tapered down so that both ZDWs [depicted by solid lines in Figs. 7(a) and (c)] decreases until they join each other at 6 $\mathrm{m}$. After this point, the fiber has all normal GVD all over the spectral range of interest here. The spectral dynamics, displayed in Figs. 77a) and (c) for simulations and experiments respectively, show the initial propagation of a soliton until $5.5 \mathrm{~m}$, where it crosses the second ZDW and thus enters the normal GVD region. a this point, a spectacular spectral broadening occurs, indicating that the pulse experiences a nonlinear. In fact, as long as the soliton spectrum starts to cross the ZDW, the emission of a dispersive wave is initiated. Since the second ZDW keeps decreasing and crossing the soliton, there is a continuous emission of radiation into the dispersive wave. Because the GVD properties of the fiber continuously changes, the phase-matching relation 5 linking the soliton and the dispersive wave continuously changes too, resulting in the generation of a broad radiation termed polychromatic dispersive wave [36, 37].

The process is as spectacular in the time domain plot of Fig. 7 (b), where it can be seen that the significant broadening occurring at around $6 \mathrm{~m}$ is accompanied by a strong temporal broadening, which is consistent with the generation of a dispersive, strongly chirped pulse. This was confirmed experimentally by recording autocorrelation traces before and after the tapered section. At $4.5 \mathrm{~m}$ [Fig. 7(e)], the pulse has a duration of $113 \mathrm{fs}$ (blue markers) and is well fitted by a square hyperbolic secant function, in good agreement with the simulation result (red solid line), which confirms its solitonic nature. But soon after the tapered section, at $6.5 \mathrm{~m}$, the autocorrelation trace is much longer and has a much distorted profile, again in agreement with simulations. At this point, there is no clue of the presence of a soliton, which has therefore totally been annihilated into a polychromatic dispersive wave.

\section{Generation of a dispersive wave continuum}

In this section, we will investigate a complex scenario in which multiple solitons and dispersive waves are generated in an axially-varying fiber. This results in the generation of a $500 \mathrm{~nm}$ supercontinuum exclusively composed of dispersive waves.

For that, we use the same configuration as in section 5 except that we increase the peak power of the input pulse to $380 \mathrm{~W}$. This exceed the required power to form a fundamental soliton given by Eq. 3 . In this case, the input pulse excites a higherorder soliton which immediately breaks up into several fundamental solitons [38]. Figures 8 a) and (b) show respectively the simulated and experimental spectral dy- 

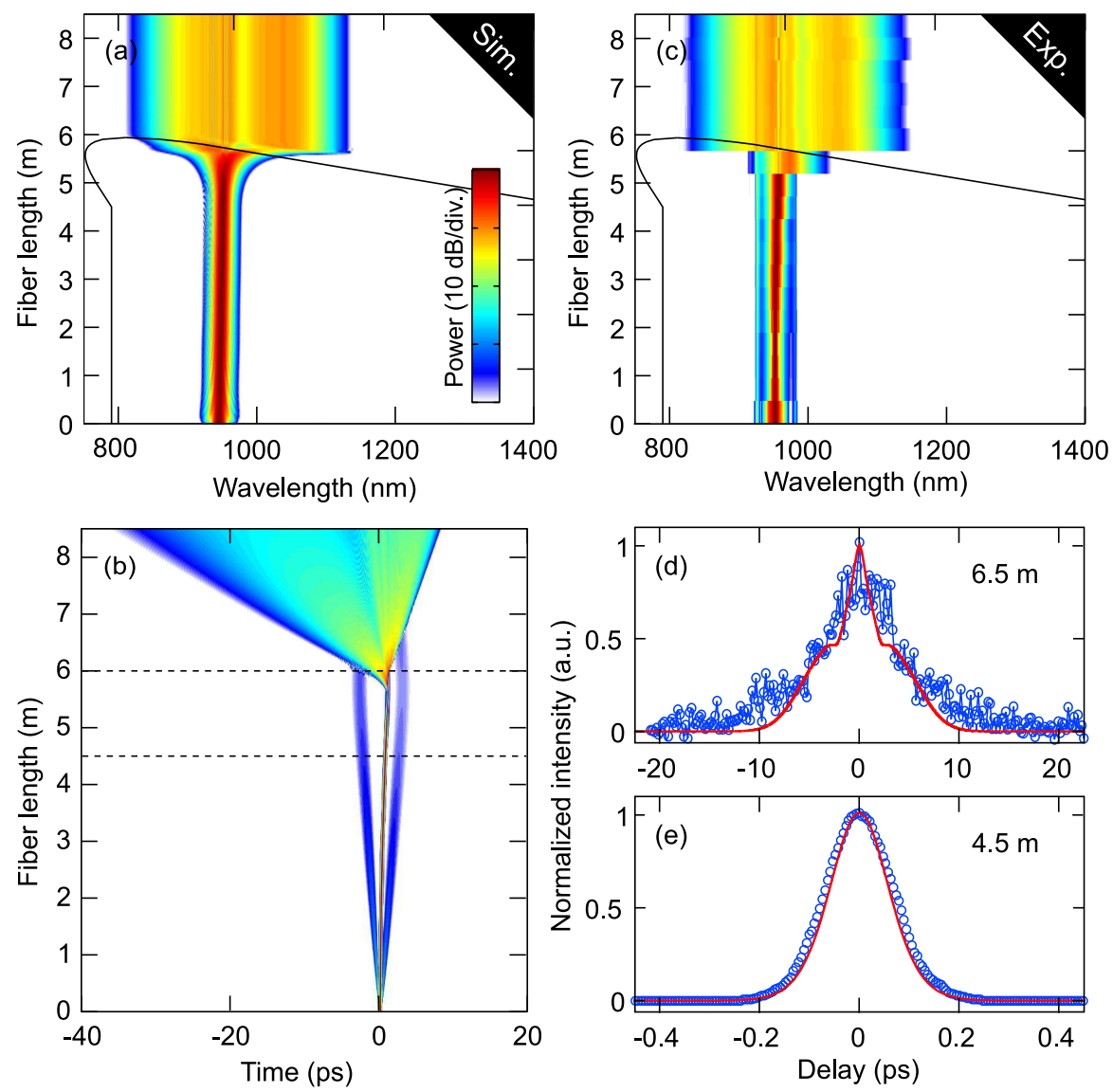

Fig. 7 (a,b) Numerical simulations and (c,d,e) experimental results showing the annihilation of a fundamental soliton into a polychromatic dispersive wave. (a) and (b) correspond respectively to the simulated spectral and temporal evolutions. (c) Measured spectral evolution versus fiber length. $(\mathrm{d}, \mathrm{e})$ Measured autocorrelation trace (open blue circles) and simulated ones (red solid line) at fiber length of (e) $4.5 \mathrm{~m}$ and (d) $6.5 \mathrm{~m}$. Black solid lines in (a) and (c) depict the ZDWs. Dashed lines in (b) depict the limits of the tapered section.

namics recorded in this case. Three main solitons, labelled $S_{1}$ to $S_{3}$, form here. The first one, $S_{1}$, is also the most powerful one [39] and therefore experiences the most efficient Raman-induced frequency shift. It rapidly hits the second ZDW (in black solid line) so that the frequency shift stops and a strong dispersive wave (labelled $\left.D W_{1}\right)$ is emitted around $1600 \mathrm{~nm}$. The soliton is frequency-locked around $1400 \mathrm{~nm}$ and hits the ZDW which starts to decrease at around $7 \mathrm{~m}$. At this point, it emits a polychromatic dispersive wave (labelled $P D W_{1}$ ) following the process described in section . The second soliton $\left(S_{2}\right)$ experiences a less significant Raman-induced selffrequency shift than $S_{1}$ and hits the ZDW around $8 \mathrm{~m}$, where it is already decreasing. Therefore, it emits a broad polychromatic dispersive wave (labelled $P D W_{2}$ ). Soliton 
$S_{3}$ remains relatively far from the ZDW all over the propagation and thus does not contribute significantly to the emission of dispersive waves.

Finally, the output spectrum between $1200 \mathrm{~nm}$ and $1600 \mathrm{~nm}$ is exclusively composed of dispersive waves and polychromatic dispersive waves generated from the two most powerful solitons.

\section{Conclusion and perspectives}

The perturbation of a fundamental soliton by third-order dispersion in optical fibers causes the emission of a resonant dispersive wave across the zero dispersion point. In axially-varying fibers, the guiding properties can be tailored as a function of propagation distance so that the ZDW continuously evolves as the soliton propagates. This allows to observe specific dispersive waves dynamics, such as the emission of cascaded, multiple or polychromatic dispersive waves.

Such axially-varying fibers can also be used to control the properties of the soliton itself, such as harnessing its Raman-induced redshift [40, 41] or even induce a blueshift [42]. In periodic fibers, multiple quasi phase matched dispersive waves can also be observed thanks to the periodicity [43, 44], following a completely different
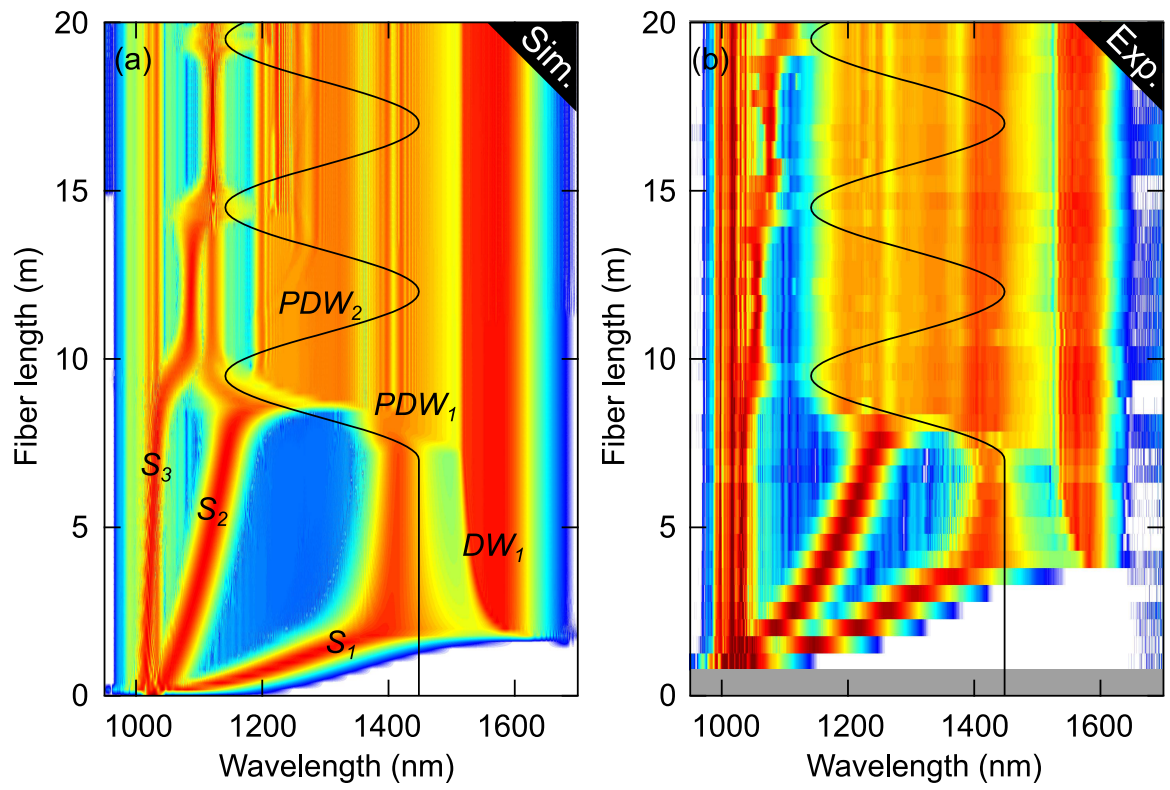

Fig. 8 (a) Numerical simulations and (b) experimental results in the spectral domain versus fiber length showing the generation of a dispersive wave continuum in an axially-varying fiber with two ZDWs. The evolution of the second ZDW (located at long wavelengths) with fiber length is represented by black solid lines. 
mechanism than the one presented here [43]. More generally, this work illustrates to remarkable robustness of fundamental solitons against various types of perturbations, and the extremely rich nonlinear dynamics that these perturbations can induce.

\section{Acknowledgements}

We acknowledge Benoît Barviau, Abdelkrim Bendahmane, Maximilien Billet, Flavie Braud, Andy Cassez, Tomy Marest, Stefano Trillo and Shaofei Wang.

\section{References}

1. N. J. Zabusky and M. D. Kruskal, "Interaction of "Solitons" in a Collisionless Plasma and the Recurrence of Initial States," Phys. Rev. Lett. 15, 240-243 (1965).

2. C. S. Gardner, J. M. Greene, M. D. Kruskal, and R. M. Miura, "Method for Solving the Korteweg-deVries Equation,” Physical Review Letters 19, 1095-1097 (1967). https://journals-aps-org.buproxy.univ-lille1.fr/prl/pdf/10.1103/PhysRevLett.19.1095 https://link.aps.org/doi/10.1103/PhysRevLett.19.1095

3. M. J. Ablowitz, D. J. Kaup, A. C. Newell, and H. Segur, "Nonlinear-Evolution Equations of Physical Significance," Physical Review Letters 31, 125-127 (1973).

https://journals-aps-org.buproxy.univ-lille1.fr/prl/pdf/10.1103/PhysRevLett.31.125 https://link.aps.org/doi/10.1103/PhysRevLett.31.125

4. V. E. Zakharov and A. B. Shabat, "Exact Theory of Two-dimensional Self-focusing and Onedimensional Self-modulation of Waves in Nonlinear Media," Soviet Journal of Experimental and Theoretical Physics 34, 62 (1972). http://adsabs.harvard.edu/abs/1972JETP...34...62Z

5. L. F. Mollenauer, R. H. Stolen, and J. P. Gordon, "Experimental Observation of Picosecond Pulse Narrowing and Solitons in Optical Fibers," Phys. Rev. Lett. 45, 1095-1098 (1980). http://link.aps.org/doi/10.1103/PhysRevLett.45.1095

6. A. Hasegawa and M. Matsumoto, Optical Solitons in Fibers (Springer-Verlag Berlin and Heidelberg GmbH \& Co. K, Berlin ; New York, 2002), dition : 3rd, revised and enlarged ed. 2003 edn.

7. J. M. Dudley, G. Genty, and S. Coen, "Supercontinuum generation in photonic crystal fiber," Rev. Mod. Phys. 78, 1135-1184 (2006). http://link.aps.org/doi/10.1103/RevModPhys.78.1135

8. Y. S. Kivshar and B. A. Malomed, "Dynamics of solitons in nearly integrable systems," Reviews of Modern Physics 61, 763-915 (1989).

https://journals-aps-org.buproxy.univ-lille1.fr/rmp/pdf/10.1103/RevModPhys.61.763 https://link.aps.org/doi/10.1103/RevModPhys.61.763

9. J. N. Elgin, "Perturbations of optical solitons," Phys. Rev. A 47, 4331-4341 (1993). http://link.aps.org/doi/10.1103/PhysRevA.47.4331

10. S. K. Turitsyn, B. G. Bale, and M. P. Fedoruk, "Dispersion-managed solitons in fibre systems and lasers," Physics Reports 521, 135-203 (2012). http://www.sciencedirect.com/science/article/pii/S0370157312002657

11. K. Hizanidis, B. A. Malomed, H. E. Nistazakis, and D. J. Frantzeskakis, "Stabilizing soliton transmission by third-order dispersion in dispersion-compensated fibre links," Pure Appl. Opt. 7, L57 (1998). http://stacks.iop.org/0963-9659/7/i=4/a=003 
12. P. K. A. Wai, C. R. Menyuk, Y. C. Lee, and H. H. Chen, "Nonlinear pulse propagation in the neighborhood of the zero-dispersion wavelength of monomode optical fibers," Opt. Lett. 11, 464-466 (1986). http://ol.osa.org/abstract.cfm?URI=ol-11-7-464

13. P. K. A. Wai, C. R. Menyuk, H. H. Chen, and Y. C. Lee, "Soliton at the zero-group-dispersion wavelength of a single-model fiber," Opt. Lett. 12, 628-630 (1987). http://ol.osa.org/abstract.cfm?URI=ol-12-8-628

14. N. Akhmediev and M. Karlsson, "Cherenkov radiation emitted by solitons in optical fibers," Phys. Rev. A 51, 2602-2607 (1995). http://link.aps.org/doi/10.1103/PhysRevA.51.2602

15. I. Cristiani, R. Tediosi, L. Tartara, and V. Degiorgio, "Dispersive wave generation by solitons in microstructured optical fibers," Optics Express 12, 124-135 (2004). http://www.opticsexpress.org/abstract.cfm?URI=oe-12-1-124

16. M. Erkintalo, Y. Q. Xu, S. G. Murdoch, J. M. Dudley, and G. Genty, "Cascaded Phase Matching and Nonlinear Symmetry Breaking in Fiber Frequency Combs," Phys. Rev. Lett. 109, 223904 (2012). http://link.aps.org/doi/10.1103/PhysRevLett.109.223904

17. K. E. Webb, Y. Q. Xu, M. Erkintalo, and S. G. Murdoch, "Generalized dispersive wave emission in nonlinear fiber optics," Opt. Lett. 38, 151-153 (2013). http://ol.osa.org/abstract.cfm?URI=ol-38-2-151

18. M. Conforti and S. Trillo, "Dispersive wave emission from wave breaking," Opt. Lett. 38, 3815-3818 (2013). http://ol.osa.org/abstract.cfm?URI=ol-38-19-3815

19. D. V. Skryabin and A. V. Gorbach, "Colloquium: Looking at a soliton through the prism of optical supercontinuum,” Rev. Mod. Phys. 82, 1287-1299 (2010). http://link.aps.org/doi/10.1103/RevModPhys.82.1287

20. A. V. Yulin, D. V. Skryabin, and P. S. J. Russell, "Four-wave mixing of linear waves and solitons in fibers with higher-orderdispersion," Opt. Lett. 29, 2411-2413 (2004). http://ol.osa.org/abstract.cfm?URI=ol-29-20-2411

21. A. Efimov, A. V. Yulin, D. V. Skryabin, J. C. Knight, N. Joly, F. G. Omenetto, A. J. Taylor, and P. Russell, "Interaction of an Optical Soliton with a Dispersive Wave," Phys. Rev. Lett. 95, 213902 (2005). http://link.aps.org/doi/10.1103/PhysRevLett.95.213902

22. D. V. Skryabin and A. V. Yulin, "Theory of generation of new frequencies by mixing of solitons and dispersive waves in optical fibers," Phys. Rev. E 72, 016619 (2005). http://link.aps.org/doi/10.1103/PhysRevE.72.016619

23. A. V. Gorbach, D. V. Skryabin, J. M. Stone, and J. C. Knight, "Four-wave mixing of solitons with radiation and quasi-nondispersive wave packets at the short-wavelength edge of a supercontinuum," Opt. Express 14, 9854-9863 (2006). http://www.opticsexpress.org/abstract.cfm?URI=oe-14-21-9854

24. A. V. Gorbach and D. V. Skryabin, "Light trapping in gravity-like potentials and expansion of supercontinuum spectra in photonic-crystal fibres," Nat Photon 1, 653-657 (2007). http://www.nature.com/nphoton/journal/v1/n11/abs/nphoton.2007.202.html

25. B. H. Chapman, J. C. Travers, S. V. Popov, A. Mussot, and A. Kudlinski, "Long wavelength extension of CW-pumped supercontinuum throughsoliton-dispersive wave interactions," Opt. Express 18, 24 729-24734 (2010). http://www.opticsexpress.org/abstract.cfm?URI=oe-18-24-24729

26. G. Agrawal, Nonlinear Fiber Optics, Fifth Edition (Academic Press, Amsterdam, 2012), 5 edition edn.

27. J. R. Taylor and R. Arguello, Roger J., "Optical Solitons Theory and Experiment," Opt. Eng 35, 2437-2439 (1996). http://dx.doi.org/10.1117/1.600816

28. L. F. Mollenauer and K. Smith, "Demonstration of soliton transmission over more than 4000 $\mathrm{km}$ in fiber with loss periodically compensated by Raman gain," Optics Letters 13, 675 (1988). https://www.osapublishing.org/ol/abstract.cfm?uri=ol-13-8-675 
29. F. R. Arteaga-Sierra, C. Milin, I. Torres-Gmez, M. Torres-Cisneros, A. Ferrando, and A. Dvila, "Multi-peak-spectra generation with Cherenkov radiation in a non-uniform single mode fiber," Opt. Express 22, 2451-2458 (2014). http://www.opticsexpress.org/abstract.cfm?URI=oe-22-3-2451

30. M. Billet, F. Braud, A. Bendahmane, M. Conforti, A. Mussot, and A. Kudlinski, "Emission of multiple dispersive waves from a single Raman-shifting soliton in an axially-varying optical fiber," Opt. Express 22, 25 673-25 678 (2014). http://www.opticsexpress.org/abstract.cfm?URI=oe-22-21-25673

31. D. Hollenbeck and C. D. Cantrell, "Multiple-vibrational-mode model for fiber-optic Raman gain spectrum and response function," J. Opt. Soc. Am. B 19, 2886-2892 (2002). http://josab.osa.org/abstract.cfm?URI=josab-19-12-2886

32. D. V. Skryabin, F. Luan, J. C. Knight, and P. S. J. Russell, "Soliton Self-Frequency Shift Cancellation in Photonic Crystal Fibers," Science 301, 1705-1708 (2003). http://www.sciencemag.org/content/301/5640/1705

33. F. Biancalana, D. V. Skryabin, A. V. Yulin, and web support@bath.ac.uk, "Theory of the soliton self-frequency shift compensation by the resonant radiation in photonic crystal fibers," Physical Review E 70 (2004). http://opus.bath.ac.uk/8984/

34. A. Bendahmane, F. Braud, M. Conforti, B. Barviau, A. Mussot, and A. Kudlinski, "Dynamics of cascaded resonant radiations in a dispersion-varying optical fiber," Optica 1, 243 (2014). https://www.osapublishing.org/optica/abstract.cfm?uri=optica-1-4-243

35. F. Braud, M. Conforti, A. Cassez, A. Mussot, and A. Kudlinski, "Solitonization of a dispersive wave," Opt. Lett., OL 41, 1412-1415 (2016). https://www.osapublishing.org/abstract.cfm?uri=ol-41-7-1412

36. C. Milin, A. Ferrando, and D. V. Skryabin, "Polychromatic Cherenkov radiation and supercontinuum in tapered optical fibers," J. Opt. Soc. Am. B 29, 589-593 (2012). http://josab.osa.org/abstract.cfm?URI=josab-29-4-589

37. A. Kudlinski, S. F. Wang, A. Mussot, and M. Conforti, "Soliton annihilation into a polychromatic dispersive wave," Optics Letters 40, 2142 (2015).

https://www.osapublishing.org/ol/abstract.cfm?uri=ol-40-9-2142

38. P. Beaud, W. Hodel, B. Zysset, and H. Weber, "Ultrashort pulse propagation, pulse breakup, and fundamental soliton formation in a single-mode optical fiber," IEEE Journal of Quantum Electronics 23, 1938-1946 (1987).

39. J. K. Lucek and K. J. Blow, "Soliton self-frequency shift in telecommunications fiber," Phys. Rev. A 45, 6666-6674 (1992). https://link.aps.org/doi/10.1103/PhysRevA.45.6666

40. A. C. Judge, O. Bang, B. J. Eggleton, B. T. Kuhlmey, E. C. Mgi, R. Pant, and C. M. de Sterke, "Optimization of the soliton self-frequency shift in a tapered photonic crystal fiber," J. Opt. Soc. Am. B 26, 2064-2071 (2009). http://josab.osa.org/abstract.cfm?URI=josab-26-11-2064

41. A. Bendahmane, O. Vanvincq, A. Mussot, and A. Kudlinski, "Control of the soliton selffrequency shift dynamics using topographic optical fibers," Opt. Lett. 38, 3390-3393 (2013). http://ol.osa.org/abstract.cfm?URI=ol-38-17-3390

42. S. P. Stark, A. Podlipensky, and P. S. J. Russell, "Soliton Blueshift in Tapered Photonic Crystal Fibers," Phys. Rev. Lett. 106, 083903 (2011). http://link.aps.org/doi/10.1103/PhysRevLett.106.083903

43. M. Conforti, S. Trillo, A. Mussot, and A. Kudlinski, "Parametric excitation of multiple resonant radiations from localized wavepackets," Scientific Reports 5, srep09 433 (2015). https://www.nature.com/articles/srep09433

44. M. Conforti, S. Trillo, A. Kudlinski, and A. Mussot, "Multiple QPM Resonant Radiations Induced by MI in Dispersion Oscillating Fibers," IEEE Photonics Technology Letters 28, 740743 (2016). 